\title{
Back to Basics, Again and Again and Again: A Longitudinal Investigation of the Effects Problem-based Tutorial Sessions Have on Student Learning Outcomes
}

\author{
George P. Banky \\ Science, Technology, Engineering and Mathematics Education Research Group, Faculty of Science, Engineering and Technology, \\ Swinburne University of Technology Melbourne, Victoria, Australia
}

Copyright $(\mathrm{C} 2018$ by authors, all rights reserved. Authors agree that this article remains permanently open access under the terms of the Creative Commons Attribution License 4.0 International License

\begin{abstract}
Engineering tertiary courses that reflect professional activity mandate the successful application of theorems and concepts in order to solve technical problems. This graduate skill is typically assessed during student problem-solving activities. Furthermore, both collaborative learning and the interactive study method known as self-explanation have been identified, by researchers, as techniques that enhance student learning. In the reported longitudinal study, students shared their solutions to problem-solving exercises with a version of self-explanation in a collaborative environment. The research investigated the effects of the intervention on student outcomes as reflected in their assessment results. In 2013, the weekly tutorial content, for a first-year electronics course, was reworked with a focus on problem-solving activities. During these sessions the students were engaged by asking them to share electronically, or via a document camera, their prepared solutions to problems that should have been attempted prior to attending. The data collected included the scores these students obtained for each of the assessable components for the course in semester one 2013, 2014, 2016 and semester two in 2016 . This data were analyzed by comparing results with those of the 2012 cohort. Week-by-week tutorial attendances showed similar trending in all semesters. The mean total mark, mean exam mark and the mean laboratory mark consistently maintained improvement over those of 2012; while the mean tutorial participation mark and the mean assignment mark were consistently worse (except for the 2014 cohort) than the corresponding ones for 2012.
\end{abstract}

Keywords Problem-solving, Self-explanation, Classroom Technology

\section{Introduction}

Researchers have identified that active collaborative learning, as well as membership in learning communities, engage learners [1]. Students have a high regard for collaboration as it enables them "to observe other students solving problems and to receive prompt feedback on misconceptions" [2, p. 10]. Furthermore, there is evidence "that active and collaborative learning techniques enhance student learning ... [and] reduce attrition" [3, p. 941].

Chi's [4] PACI (passive-active-constructive-interactive) theoretical framework was the catalyst for the intervention described in this paper. In the acronym PACI, Chi identifies four learning styles in increasing order of effectiveness, with the interactive style incorporating the intellectual mechanisms of both constructive and active learning [5]. In this study the research focused on investigating communal interactive learning during tutorials for a first-year electronics systems course at an Australian university.

While demonstrating their own attempts to solve the previously set problems, the students were given the opportunity to explain their solutions to others in a tutorial venue. This is a variation on the "self-explanation" technique [6] where the explanation is not just for one's benefit, but also for the benefit of students who were in attendance at the sessions. A subsequent adoption of this learning strategy could be of great benefit to the students, as evidenced by "research across a variety of domains [which] has consistently supported the findings that students learn better when they explain to themselves [or to others] the material they are studying" [5, p. 296]. 


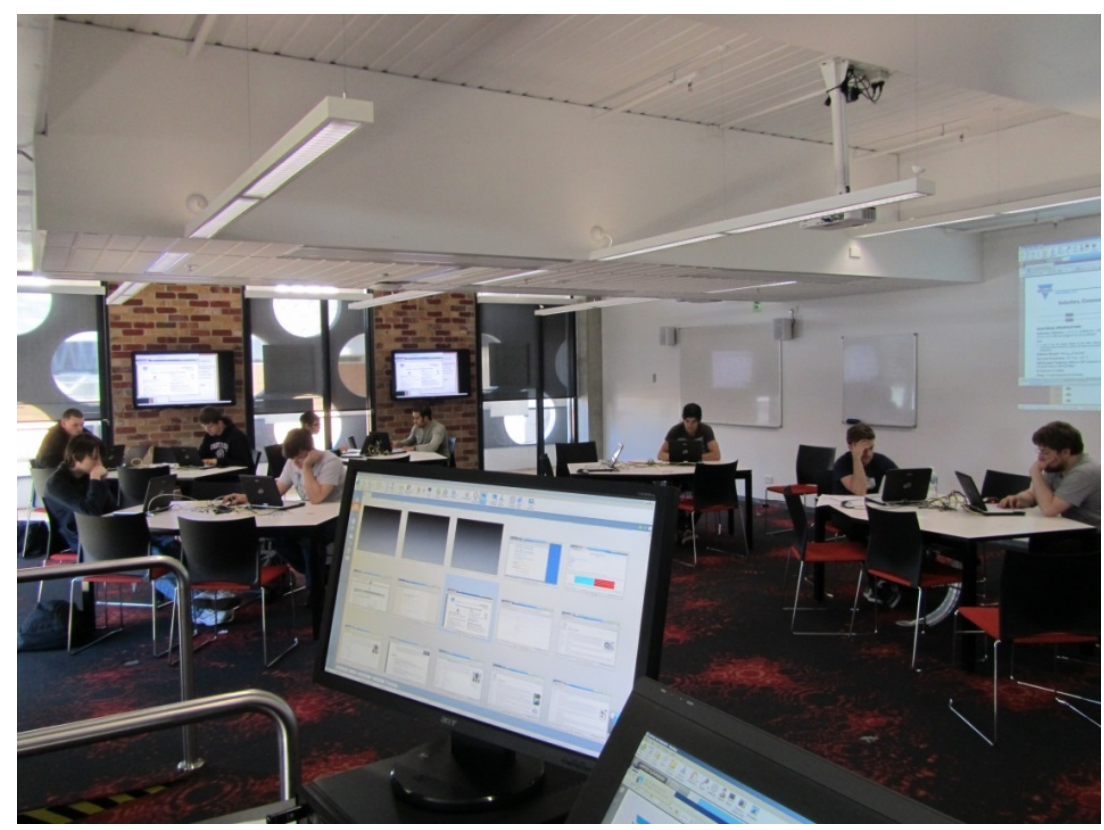

Figure 1. Venue used for NetSupport School investigation

In this four-academic-semesters-long study (the first semester's results were reported at the 2013 Australasian Association for Engineering Educators (AAEE) conference [7]) a local-area network-based collaboration software, NetSupport School

(http://www.netsupportsoftware.com), was used to establish a co-operative environment in the computer laboratory that was used as the teaching space. The ensuing setup directly supported the real-time supervision of student activities with virtual "teaching between desks" (that is also known as "kikan-shido" [8]) and over-the-shoulder learning and teaching (OTST/L) [9]. These pedagogies have been identified as being dominantly present in laboratories and tutorials [10]. Without such a software application, implementing physical "kikan-shido" and OTST/L supervision of student learning, particularly with computer-screen-based activity on tablet computers would have been extremely difficult. Only tablet computers were available in the venue during two of the observed semesters.

\section{Purpose}

The research question addressed by this study was:

- Will problem-solving exercises attempted by students before their tutorials, followed by the opportunity to collaboratively participate in a learning community (such as tutorials where the students demonstrate and explain their solutions), result in better learning outcomes?

\section{Design/Method}

The venue chosen for the reported research and shown in Figure 1, had twenty computers. A copy of NetSupport School was installed on each of the venue's machines. This software facilitated the monitoring, by an academic, in real-time over a local-area network, the on-screen activities of the students working in this computer laboratory.

On a desk, at the front of the room, the academic had a dual-screen desktop computer. One monitor was dedicated to displaying the image that was showing on the video projectors and the video screens, which were placed around the walls of the room. The other screen displayed the NetSupport School control window (as shown in Figure 2) facilitating, in real-time, the viewing of the thumbnails of the students' on-screen activities.

The academic, by double-clicking on any of the displayed thumbnails, could connect with the displayed computer so the two computers behaved as if they were one: the remote screen filled one of the academic's desktop computer's monitor; and the remote machine could be controlled by that desktop's keyboard and mouse. Furthermore, by dragging the window showing the student's work to the desktop's other display monitor, this image may be shown on the video screens and projector images around the room, thereby presenting it to the rest of the attendees for discussion.

The participants selected for this investigation, were first-year undergraduate engineering students who were studying electronic systems for one semester of their engineering program at Swinburne University of Technology. In 2012, 2013, 2014 and 2016, between eleven and thirteen tutorial classes per week were timetabled for this course; each with no more than eighteen students per session. 


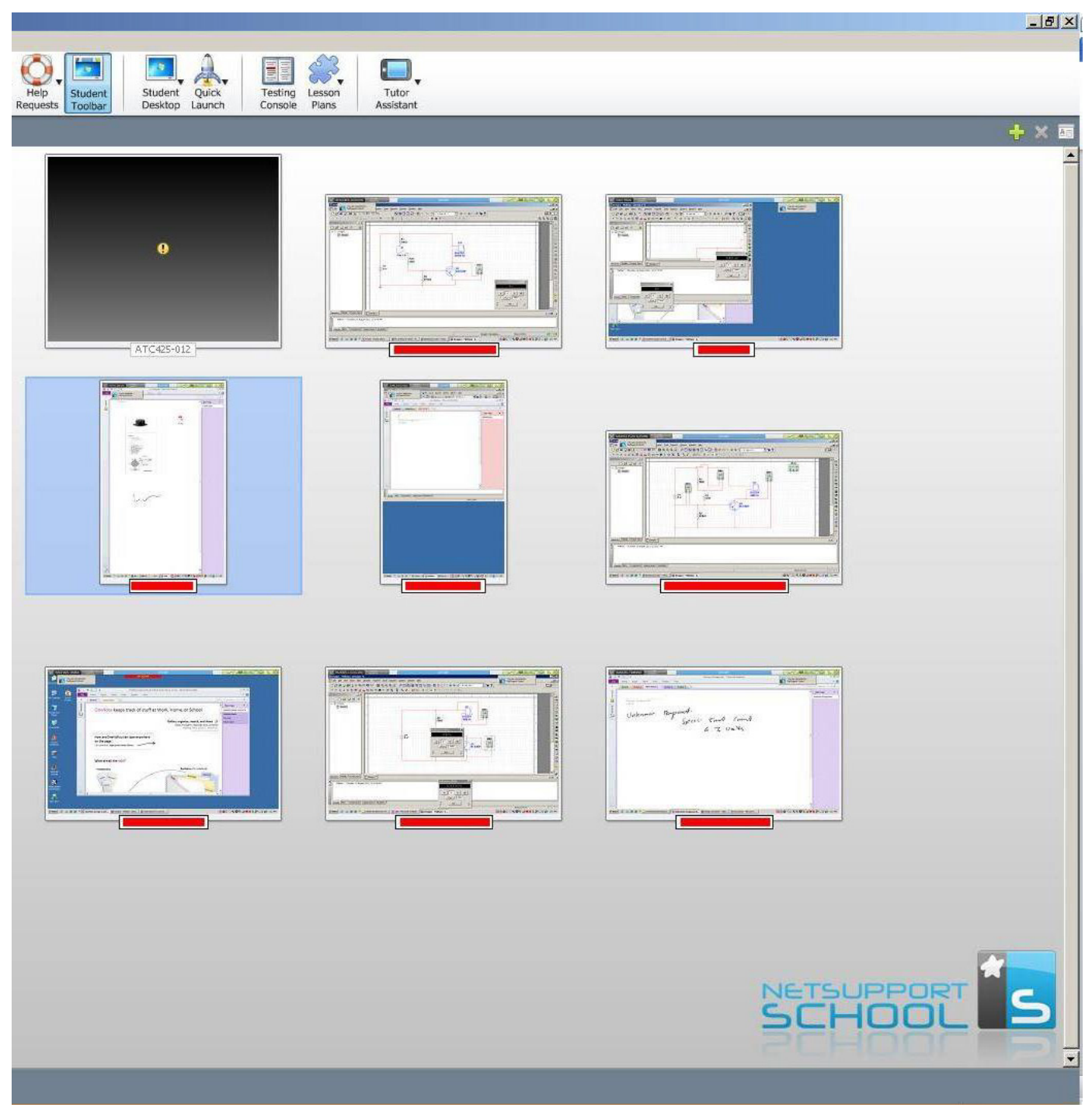

Figure 2. NetSupport School control screen displaying real time "thumbnails" of the connected students' on-screen activities

In an attempt to minimize research bias, the course syllabus, the laboratory experiments, the assignment problems, the questions of the closed-book exam (previous exams were unavailable to the students), and the problems for discussion in each corresponding tutorial were identical in each of the investigated semesters. The same academic delivered the lectures and the same pair of demonstrators supervised the laboratory experiments during the study. Furthermore, the one tutor was involved with all tutorials in 2012, 2013 and 2014; while in 2016 he shared the groups with another tutor who familiarized himself with the delivery technique, by attending a tutorial conducted by the first tutor before starting any of his own sessions.

From 2013 onwards, the tutorial activities for the course involved only pre-attendance problem solving and subsequent solution sharing by the attending students. The fundamental change introduced in 2013 was the requirement for students to prepare for tutorial sessions with documented attempts at solving the problems which were to be discussed during the corresponding tutorials.
This preliminary work was evidenced by the student uploading an electronic copy of their solutions onto the university's course management system, Blackboard (http://www.blackboard.com/), prior to attending their scheduled sessions. The associated Blackboard course upload portal was customized for each student to open after their tutorial ended and close before their next tutorial started; giving them one academic week to submit. Then, during the corresponding tutorial each student was asked to contribute by sharing their detailed solution (from the screen of their computer or via a document camera at the front the room) or describing the relevant theorems they have used in attempting the exercise of solving a number of electronic circuit analysis problems. The students' tutorial participation mark was a " 1 " if they uploaded an attempt, attended the tutorial and contributed to the proceedings; otherwise it was a " 0 ". The 2012 marking scheme gave the student a " 1 " if they attended and a " 0 " if they did not attend the session. 


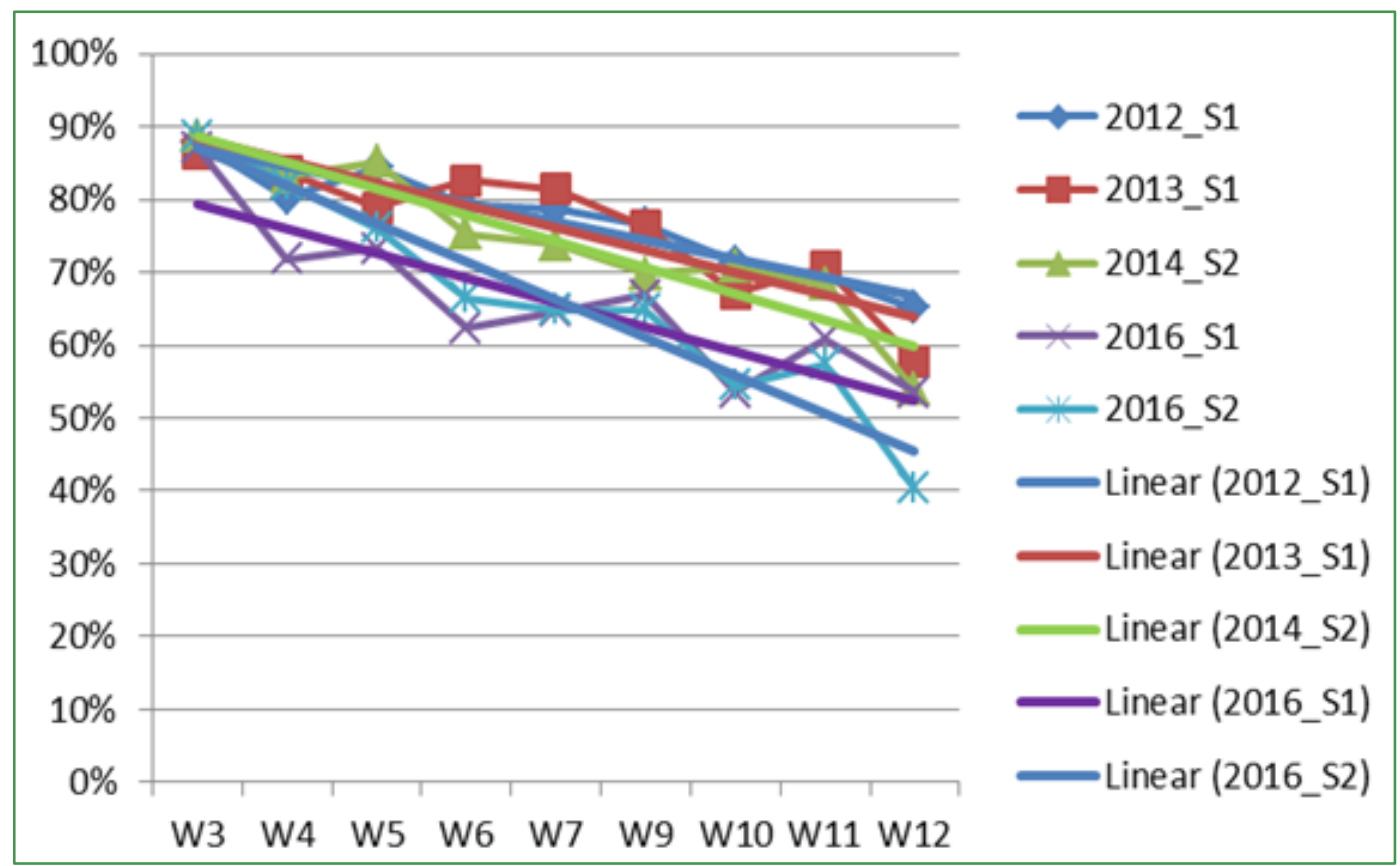

Figure 3. Percentage of enrolled students who attended each of the tutorials from Academic Week 3 (W3) to Academic Week 7 (W7) and from Academic Week 9 (W9) to Academic Week 12 (W12)

Table 1. Percentage changes in average (mean) marks over those for semester one of 2012

\begin{tabular}{|c|c|c|c|c|c|}
\hline Year & 2016_S2 & 2016_S1 & 2014_S1 & 2013_S1 & 2012_S1 \\
\hline Completed Student Numbers & 121 & 175 & 172 & 145 & 168 \\
\hline (Mean Exam Mark) $/ 60$ & $9.9 \%$ & $8.2 \%$ & $15.5 \%$ & $14.1 \%$ & \\
\hline (Mean Assignment Mark) $/ 15$ & $-10.7 \%$ & $-11.0 \%$ & $9.0 \%$ & $-9.0 \%$ & \\
\hline (Mean Tutorial Participation Mark) $/ 5$ & $-32.5 \%$ & $-30.7 \%$ & $-14 . .4 \%$ & $-8.1 \%$ & \\
\hline (Mean Laboratory Participation Mark) $/ 20$ & $28.6 \%$ & $28.1 \%$ & $40.7 \%$ & $28.9 \%$ & \\
\hline (Total Mark)/100 & $7.6 \%$ & $7.6 \%$ & $16.8 \%$ & $12.0 \%$ & \\
\hline
\end{tabular}

Five assignments (one every two weeks) requiring the application of the previous fortnight's lecture topics, were also a required assessed submission from each student during their semester. One 2 hours long, end-of-semester, closed-book examination; and laboratory participation that reflected preliminary work, attendance and one formal report completed their assessments each semester. The criteria for all assessable components, except for the tutorial participation, have remained constant during all the semesters forming this study.

Historically, the use of student marks for educational research has been a controversial one [11]. However, it is the opinion of this researcher that for this research feedout, rather than feedback, assessment was required in order to comparatively reflect on the students' abilities with respect to assessed outcomes, which ideally should also be the stated ones for the course [12].

\section{Results}

Figure 3 shows a graph of the percentage of enrolled students who attended the tutorials in Academic Week 3 to Academic Week 7, and Academic Week 9 to Academic Week 12, in the first semesters of 2012, 2013, 2014 and 2016 as well as in the second semester of 2016. As can be seen in Figure 3, there were no tutorials held in Academic Week 8 of the first semesters, because a significant number of the classes were scheduled on Anzac Day (a public holiday in Australia).

Table 1 displays the percentage changes in the average marks obtained, for each assessed component, by the participating students, over the four semesters under investigation, with respect to semester 1 in 2012 (2012_S1).

\section{Discussion}

Figure 3 shows the percentages of students who attended their tutorial sessions each week in the first semesters of 2012, 2013, 2014 and both semesters of 2016. The data trends (represented by the five linear graphs) visually highlight the fact that the trending for each semester is 
significantly similar. Anecdotally, very similar trending appears to describe the tutorial attendance percentages for most, if not all, engineering courses at all levels in Australian tertiary institutions. Furthermore, as seen in Figure 3, for the actual percentages, there was an initial settling period (until Academic Week 5), that was followed by a continuing decline in the numbers who attended. Since the course that was selected for this study was a first-year one, one must consider student transitioning issues to have an effect on the collected data.

As seen in Table 1, the percentage changes in the mean marks obtained by the cohorts for their assessable material, shows that in 2013, 2014 and 2016 (when compared to those of 2012) the mean of the exam marks improved by between $8.2 \%$ and $14.1 \%$, the mean laboratory participation mark by between $28.1 \%$ and $40.7 \%$, and the mean total mark by between $7.60 \%$ and $16.8 \%$. While the mean tutorial participation mark dropped by between $8.1 \%$ and $32.5 \%$, and the mean assignment mark by between $9 \%$ and $11.0 \%$ except for 2014 when it improved by $9 \%$. The worsened mean tutorial participation marks reflect the changed criteria in the implemented marking scheme, namely: one mark if there was an upload attempt prior to the session plus attendance plus contribution at the session as compared to one mark just for attending the session.

The observable similarity in the trending for tutorial attendance (as shown by the linear graphs in Figure 3) indicates, that even though there was a change to the criteria used to award participation marks, the students who attended without preparation may also have benefited from just being present at the tutorial sessions. The improved means of the other assessable components indicate that there may have been some beneficial effect on the outcomes for these onlookers, or "lurkers", as well.

The worsened mean assignment marks possibly reflect the extra time needed for the preparation of the tutorial attempts, hence causing less time for the students to complete their fortnightly assignments.

The 2014 cohort appears to have excelled in all the metrics while their tutorial attendances trended with those of the other years. This may imply that overall they may have been an academically better group of students. Conversely, the 2016 groups performed worse than the previous years' cohorts. This may be indicative of the fact, that since the course was going to be discontinued after 2016, a greater number of repeating students (with potentially lower levels of academic skills) were enrolled in the last year that the course was offered.

In both semesters of 2016, two tutors were involved in content delivery; each tutor taking approximately half the sessions in each semester. The results for 2016 indicate that the percentage changes (with respect to those of 2012) for the obtained mean marks trended the same as for the other years when the same tutor took all the tutorials. An explanation for this observation may be that student outcomes resulting from the application of the "attempt-submission-followed-by-group-explanation" style of tutorials were tutor-independent.

While the mean exam outcomes generally improved, since not all students availed themselves of the tutorials on offer, the marks of those who did not attend could have negatively impacted on the calculated mean. It then follows that by achieving higher tutorial attendances the improvement in outcomes would most likely increase.

It must be noted that the comparative analysis of the mean marks for each assessed component is not endorsing statements such as: "exam marks are a good indicators of student learning" [11], however it does probably articulates a lot about the appropriateness of the "how", the "why" and the "what" of the assessments given to the students [12]. Since the ultimate focus for engineering course assessments is on the application of concepts, understandably any additional problem-solving activities (such as those of the 2013 onward tutorials) are expected to have a positive effect on subsequent marks if the assessments were reflective of such a focus.

\section{Conclusions}

Successful collaborative learning can only occur in environments where the participants have the opportunity to engage in what they perceive to be a normal discussion [13]. In the longitudinal research reported in this paper two emerging technologies were used to establish the environment. These were the computers in a laboratory setting, and a computer collaboration utility (NetSupport School). The self-explanation effect [6] driven by mandatory preparation and collaboration with the attending students during tutorials was the likely cause for improvements in the mean exam marks of the students for solving quantitative exam problems.

There is no question that the pedagogical 'playing field' is changing by the promise of ubiquitously available low cost touch-screen based portable computing equipment for students. This must have an effect on how students record and learn educational content [14]. Furthermore, the current predictions for future developments in software simulators promise 3D and haptic-enabled interfaces that will facilitate the mimicking of realistic appearance, touch and feel of simulated objects and/or activities. Ultimately, such devices will dramatically change the delivery of experiential learning that is currently offered in face-to-face laboratory and tutorial sessions in brick-and-mortar venues; both on or off campus. In any case, it is important to note that the primary driver for selecting and subsequently using any technological advancement in educational contexts must always be pedagogy rather than technology $[15,16]$.

Finally, it is clear from the results of this research that the practice of self-explanation, even in the company of 
others, will improve the students' problem solving skills during their studies. While the reported study was conducted in a brick-and-mortar environment, the technologies mentioned above are capable of facilitating real-time collaboration that perform equally well on both local-area (LAN) and wide-area networks (WAN).

\section{Acknowledgements}

The author wishes to acknowledge: the support received from the Science, Technology, Engineering and Mathematics Education Research (STEMeD) Group within the Faculty of Science, Engineering and Technology at Swinburne University of Technology, and the in-kind contributions received from Pixel IT Pty. Ltd. (http://www.pixel.com.au) - the Australian distributor of NetSupport School, and National Instruments Australia (http://australia.ni.com).

\section{REFERENCES}

[1] Leach, L. and N. Zepke, Engaging students in learning: a review of a conceptual organiser. Higher Education Research \& Development, 2011. 30(2): p. 193-204.

[2] Donovan, D. and B. Loch, Closing the feedback loop: engaging students in large first-year mathematics test revision sessions using pen-enabled screens. International Journal of Mathematical Education in Science and Technology, 2013. 44(1): p. 1-13.

[3] Loch, B., et al., Learner-centred mathematics and statistics education using netbook tablet PCs. Journal of Mathematical Education in Science and Technology, 2011. 42(7): p. 939-949.

[4] Chi, M.T.H., Active-constructive-interactive: A conceptual framework for differentiating learning activities. Topics in Cognitive Science, 2009(I): p. 73-105.

[5] Fonseca, B.A. and M.T.H. Chi, Instruction Based on Self-explanation, in The Handbook of Research on Learning and instruction, R.E. Mayer and P.A. Alexander,
Editors. 2011, Routledge Taylor and Frances Group: New York, NY. p. 296-321.

[6] Chi, M.T.H., et al., Self-explanations: How students study and use examples in learning to solve problems. Cognitive Science, 1989. 13: p. 134-182.

[7] Banky, G.P., Back to basics: Investigating the effect of problem-based tutorial sessions on solving quantitative exam problems, in Australasian Association for Engineering Education (AAEE) Annual Conference. 2013: Gold Coast, Qld.

[8] Clarke, D.J., CHAPTER TWO: The LPS Research Design, in Mathematics Classrooms in Twelve Countries: The Insider's Perspective, D.J. Clarke, C. Keitel, and Y. Shimizu, Editors. 2006, Sense Publishers: Rotterdam, The Netherlands. p. 15-36.

[9] Twidale, M.B., Over the shoulder learning: supporting brief informal learning. Computer Supported Cooperative Work: The Journal of Collaborative Computing, 2005. 14(6): p. 505-547.

[10] Banky, G.P., Looking for Kikan-Shido: Are elements of it detectable in tertiary engineering pedagogy?, in Australasian Association for Engineering Education 2007 Conference. 2007: Melbourne, Australia.

[11] Knight, P.T., Summative Assessment in Higher Education: practices in disarray. Studies in Higher Education, 2002. 27(3): p. 275-286

[12] Martin, F. and A. Ndoye, Using Learning Analytics to Assess Student Learning in Online Courses. Journal of University Teaching \& Learning Practice, 2016. 13(3).

[13] Pincas, A., Successful online course design: Virtual frameworks for discourse construction. Educational Technology \& Society, 1998. 1(1): p. 14-25.

[14] Banky, G.P., Chapter VII: The (digital) natives are restless: Reflections from the coalface, in INNOVATIONS 2013. World Innovations in Engineering Education and Research, W. Aung, et al., Editors. 2013, iNEER: Potomac, MD. p. 71-80.

[15] Laurillard, D., The pedagogical challenges of collaborative technologies. International Journal of Computer-Supported Collaborative Learning, 2009. 4(1): p. 5-20.

[16] Ramsden, P., Learning to Teach in Higher Education. 2nd. ed. 2003, London, UK: RoutledgeFalmer. 